\title{
Self-Management Education Class Attendance and Health Care Provider Counseling for Physical Activity Among Adults with Arthritis - United States, 2019
}

\author{
Lindsey M. Duca, $\mathrm{PhD}^{1,2}$; Charles G. Helmick, $\mathrm{MD}^{2}$; Kamil E. Barbour, $\mathrm{PhD}^{2}$; Dana Guglielmo, $\mathrm{MPH}^{2,3}$; Louise B. Murphy, PhD 2 ; \\ Michael A. Boring, $\mathrm{MS}^{2}$; Kristina A. Theis, $\mathrm{PhD}^{2}$; Erica L. Odom, $\mathrm{DrPH}^{2}$; Yong Liu, $\mathrm{MD}^{2}$; Janet B. Croft, $\mathrm{PhD}^{2}$
}

Arthritis is a highly prevalent and disabling condition among U.S. adults (1); arthritis-attributable functional limitations and severe joint pain affect many aspects of health and quality of life (2). Self-management education (self-management) and physical activity can reduce pain and improve the health status and quality of life of adults with arthritis; however, in 2014, only $11.4 \%$ and $61.0 \%$ of arthritis patients reported engaging in each, respectively. To assess self-reported self-management class attendance and health care provider physical activity counseling among adults with doctor-diagnosed arthritis, CDC analyzed 2019 Behavioral Risk Factor Surveillance System (BRFSS) data. In 2019, an age-standardized state median of one in six (16.2\%) adults with arthritis reported ever attending a selfmanagement class, and $69.3 \%$ reported ever receiving health care provider counselling to be physically active. Prevalences of both differed by state and sociodemographic characteristics; decreased with lower educational attainment, joint pain severity, and urbanicity; and were lower in men than in women. Health care providers can play an important role in promoting self-management class attendance and physical activity by counseling arthritis patients about their benefits and referring patients to evidence-based programs (3).

BRFSS is an annual, cross-sectional, state-based telephone survey conducted among the noninstitutionalized U.S. population aged $\geq 18$ years. ${ }^{*}$ In 2019 , the median combined landline and cellular survey response rate for 49 states $^{\dagger}$ and the District of Columbia (DC) was 49.4\% (range $=37.3 \%-73.1 \%)$. Participants were identified as having arthritis if they responded "yes" to the question, "Have you ever been told by a doctor or other health care professional that you have arthritis, rheumatoid arthritis, gout, lupus, or fibromyalgia?" Among 135,862 adults with arthritis, self-management class attendance was defined by an affirmative response to the question, "Have you ever taken an educational course or class to teach you how to manage problems related to your arthritis or joint symptoms?" Respondents with arthritis were classified as having received

\footnotetext{
${ }^{*}$ https://www.cdc.gov/brfss/about/index.htm

$\dagger$ In 2019, New Jersey did not collect sufficient data to meet the minimum requirement for inclusion in the BRFSS public-use data set.

${ }^{\$}$ https://www.cdc.gov/brfss/annual_data/2019/pdf/2019-response-ratestable-508.pdf

https:/www.cdc.gov/arthritis/basics/types.html
}

health care provider counseling for physical activity if they answered "yes" to the question, "Has a doctor or other health professional ever suggested physical activity or exercise to help your arthritis or joint symptoms?"

Among adults with arthritis in 49 states and DC, state-specific unadjusted and age-standardized ${ }^{* *}$ prevalences (with $95 \%$ confidence intervals [CIs]) were calculated for self-management class attendance or having received health care provider counseling (counseling) to be physically active. Differences in the prevalences of these two outcomes by selected characteristics were assessed in age-adjusted ${ }^{\dagger \dagger}$ logistic regression models that included age as a categorical covariate. All analyses accounted for BRFSS's complex sampling design and sampling weights, based on iterative proportional fitting, were applied to make state-specific estimates representative of each state. ${ }^{\$ \$}$ Analyses were conducted using SAS (version 9.4; SAS Institute) and SUDAAN (version 11.0; RTI International). This activity was reviewed by CDC and was conducted consistent with applicable federal law and CDC policy. 99

In 2019, among 49 states and DC, a median of $23.6 \%$ of respondents reported having arthritis. Among adults with arthritis, the median age-standardized prevalence of reported self-management class attendance was $16.2 \%$ (range $=9.8 \%$ [DC] to $24.9 \%$ [Hawaii]) (Table 1). Age-adjusted prevalence reflected lower self-management class attendance among men $(15.4 \%)$ than among women (17.0\%), among non-Hispanic White $(15.6 \%)$ or Hispanic (17.0\%) persons than among non-Hispanic Asian (20.9\%), American Indian or Alaska Native $(21.9 \%)$, or other or multiple race $(21.2 \%)$ persons, and among those never married (15.0\%) or a member of an unmarried couple $(15.8 \%)$ than among those married (16.0\%) or divorced, separated, or widowed (17.3\%) (Table 2). Age-adjusted prevalence increased with higher educational

\footnotetext{
** Estimates were age-standardized to the 2000 U.S. Projected Population aged $\geq 18$ years using three age groups: $18-44,45-64$, and $\geq 65$ years to allow for state-to-state comparisons. https://www.cdc.gov/nchs/data/statnt/statnt20.pdf

${ }^{\dagger \dagger}$ Age-adjusted estimates were generated in weighted logistic regression models that included age as a categorical covariate with the following cut points: $18-44$ years, $45-64$ years, and $\geq 65$ years.

$\$ \$$ http://citeseerx.ist.psu.edu/viewdoc/download?doi=10.1.1.684.5837\&rep=r ep1\&type=pdf

9945 C.F.R. part 46.102(1)(2), 21 C.F.R. part 56; 42 U.S.C. Sect. 241(d); Sect. U.S.C. Sect. 552a; 44 U.S.C. Sect. 3501 et seq.
} 
TABLE 1. Unadjusted and age-standardized* prevalence of self-management education class attendance ${ }^{\dagger}$ and receipt of health care provider counseling about physical activity ${ }^{\S}$ among adults with arthritis ${ }^{\mathbb{\natural}}$ aged $\geq 18$ years - Behavioral Risk Factor Surveillance System, United States, ${ }^{* *} 2019$

\begin{tabular}{|c|c|c|c|c|c|c|c|c|c|}
\hline \multirow[b]{3}{*}{ Jurisdiction } & \multicolumn{3}{|c|}{ Persons with arthritis } & \multicolumn{3}{|c|}{$\begin{array}{c}\text { Self-management education } \\
\text { class attendance }\end{array}$} & \multicolumn{3}{|c|}{$\begin{array}{c}\text { Health care provider } \\
\text { physical activity counseling }\end{array}$} \\
\hline & \multirow[b]{2}{*}{ Est. no. ${ }^{\dagger \dagger}$} & \multicolumn{2}{|c|}{$\%(95 \% \mathrm{Cl})$} & \multirow[b]{2}{*}{ Est. no. ${ }^{\dagger \dagger}$} & \multicolumn{2}{|c|}{$\%(95 \% \mathrm{Cl})$} & \multirow[b]{2}{*}{ Est. no. ${ }^{\dagger \dagger}$} & \multicolumn{2}{|c|}{$\%(95 \% \mathrm{Cl})$} \\
\hline & & Unadjusted & $\begin{array}{l}\text { Age- } \\
\text { standardized }\end{array}$ & & Unadjusted & $\begin{array}{c}\text { Age- } \\
\text { standardized }\end{array}$ & & Unadjusted & $\begin{array}{c}\text { Age- } \\
\text { standardized }\end{array}$ \\
\hline Median, $§ \S \%$ & NA & 26.1 & 23.6 & NA & 15.7 & 16.2 & NA & 70.4 & 69.3 \\
\hline Alabama & $1,273,000$ & $33.9(32.5-35.3)$ & $30.4(29.2-31.6)$ & 191,000 & $15.1(13.5-16.9)$ & $17.3(14.4-20.7)$ & 871,000 & $69.0(66.7-71.1)$ & $69.1(65.3-72.6)$ \\
\hline Alaska & 116,000 & $1.4(19.4-23.5)$ & $20.9(19.2-22.8)$ & 23,000 & $19.8(15.7-24.6)$ & $21.1(14.7-29.2)$ & 83,000 & $72.3(68.0-76.2)$ & $75.8(69.8-80.9)$ \\
\hline Arizona & $1,301,000$ & $23.6(22.2-24.9)$ & $21.0(19.8-22.3)$ & 226,000 & $17.5(15.3-19.9)$ & $16.2(12.6-20.4)$ & 907,000 & $70.1(67.1-73.0)$ & $67.1(61.0-72.7)$ \\
\hline Arkansas & 715,000 & $31.2(29.6-32.9)$ & $28.5(27.0-30.0)$ & 104,000 & $14.6(12.6-16.8)$ & $14.7(11.6-18.5)$ & 466,000 & $66.3(63.4-69.0)$ & $63.2(58.0-68.1)$ \\
\hline California & $6,007,000$ & $19.8(18.9-20.7)$ & $18.4(17.6-19.2)$ & $1,192,000$ & $19.9(17.9-22.1)$ & $20.4(16.9-24.4)$ & 336,000 & $72.7(70.4-74.9)$ & $70.3(66.3-74.1)$ \\
\hline Colorado & 990,000 & $22.3(21.4-23.2)$ & $21.1(20.2-22.0)$ & 154,000 & $15.6(14.0-17.4)$ & $16.5(13.8-19.7)$ & 678,000 & $69.8(67.7-71.9)$ & $69.3(65.5-72.8)$ \\
\hline Connecticut & 653,000 & $23.5(22.5-24.6)$ & $20.3(19.4-21.3)$ & 78,000 & $12.0(10.6-13.6)$ & $12.8(9.8-16.5)$ & 467,000 & $72.6(70.3-74.7)$ & $71.3(66.2-75.9)$ \\
\hline Delaware & 208,000 & $27.4(25.6-29.3)$ & $23.6(22.0-25.3)$ & 33,000 & $15.6(13.2-18.4)$ & $15.4(11.8-19.7)$ & 152,000 & $73.1(69.7-76.2)$ & $69.1(62.2-75.2)$ \\
\hline $\begin{array}{l}\text { District of } \\
\text { Columbia }\end{array}$ & 97,000 & $17.2(15.7-18.9)$ & $18.7(17.3-20.3)$ & 15,000 & 15.7 (12.9-18.9) & $9.8(7.4-12.7)$ & 73,000 & $77.3(72.7-81.3)$ & $74.4(66.0-81.3)$ \\
\hline Florida & & $25.4(24.1-26.7)$ & $21.1(2$ & & $4(17.9-23.2)$ & & 00 & $-73.7)$ & $70.2(65$. \\
\hline Georgia & $1,902,000$ & $23.8(22.4-25.2)$ & $22.2(21.0$ & , 000 & 15.9 (13.7-18.4) & $17.0(12.7$ &, 000 & $67.0(63.8-70.0)$ & $63.4(57.5$ \\
\hline Hawaii & 230,000 & $20.9(19.8-22.1)$ & 18.4 (17.4-19.5) & 48,000 & $20.8(18.3-23.5)$ & $24.9(20.2-$ & 159,000 & $69.7(66.8-72.4)$ & 66.7 \\
\hline Idaho & 329,000 & $25.1(23.4-26.8)$ & $23.1(21.5-24.7)$ & 64,000 & $19.5(16.4-23.1)$ & $21.1(15.4-28.3)$ & 212,000 & $65.9(62.3-69.4)$ & $67.1(61.0-72.6)$ \\
\hline Illinois & $2,409,000$ & $24.7(23.5-26.0)$ & $22.5(21.4-23.7)$ & 415,000 & $17.2(15.2-19.5)$ & $15.8(12.9-19.2)$ & $1,715,000$ & $71.6(68.9-74.2)$ & $70.5(65.9-74.6)$ \\
\hline Indiana & $1,358,000$ & $26.9(25.9-28.0)$ & $24.7(23.7-25.7)$ & 216,000 & $16.0(14.4-17.7)$ & $16.3(13.5-19.5)$ & 921,000 & $68.8(66.6-70.9)$ & $68.0(64.2-71.6)$ \\
\hline lowa & 618,000 & $25.7(24.7-26.6)$ & $23.0(22.1-23.9)$ & 94,000 & $15.4(14.0-16.9)$ & $17.0(14.5-19.8)$ & 408,000 & $67.3(65.3-69.2)$ & $65.5(62.0-68.9)$ \\
\hline Kansa & 555,000 & $25.6(24.7-26.5)$ & $23.6(22.7-24.4)$ & 89,000 & $16.1(14.6-17.6)$ & $15.7(13$. & 374,000 & $68.6(66.6-70.5)$ & 65.7 \\
\hline Kentucky & $1,176,000$ & $34.3(32.7-35.9)$ & $31.3(29.8-32.9)$ & 157,000 & $13.4(11.5-15.4)$ & $14.0(11.3-17.0)$ & 796,000 & $68.4(65.8-70.9)$ & $66.1(61.9-70.0)$ \\
\hline Louisiana & 968,000 & $27.6(26.1-29.2)$ & $25.5(24.2-26.9)$ & 140,000 & $14.6(12.5-16.8)$ & $15.3(12.2-18.9)$ & 686,000 & $71.8(69.0-74.5)$ & $72.9(68.5-76.9)$ \\
\hline Maine & 340,000 & $31.8(30.5-33.1)$ & $27.4(26.1-28.8)$ & 48,000 & $14.1(12.6-15.7)$ & $13.7(11.2-16.8)$ & 238,000 & $71.3(69.0-73.4)$ & $70.6(66.0-74.8)$ \\
\hline Maryland & $1,107,000$ & $23.9(23.1-24.8)$ & $21.6(20.9-22.4)$ & 178,000 & $16.2(14.8-17.6)$ & $17.7(14.7-21.1)$ & 826,000 & $75.3(73.7-76.9)$ & $75.2(71.9-78.2)$ \\
\hline Massachusetts & $1,316,000$ & $24.5(23.3-25.7)$ & $21.9(20.8-23.0)$ & 205,000 & 15.7 (13.9-17.7) & $15.1(12.3-18.4)$ & 945,000 & 73.5 (71.1-75.8) & $72.0(67.6-76.0)$ \\
\hline Michigan & $2,373,000$ & $30.8(29.6-31.9)$ & $27.2(26.2-28.2)$ & 345,000 & $14.6(13.2-16.0)$ & $14.5(12.3-17.0)$ & $1,665,000$ & $71.0(69.0-72.9)$ & $70.6(66.9-74.0)$ \\
\hline Minnesota & 928,000 & $21.7(20.9-22.4)$ & $19.4(18.8-20.1)$ & 175,000 & $19.0(17.6-20.5)$ & $18.4(16.2-20.8)$ & 629,000 & $69.1(67.3-70.8)$ & $67.5(64.4-70.5)$ \\
\hline Mississippi & 650,000 & $28.8(27.3-30.4)$ & $26.3(24.9-27.7)$ & 92,000 & $14.2(12.1-16.7)$ & $18.5(13.9-24.1)$ & 442,000 & $68.7(65.8-71.5)$ & $69.5(64.6-74.0)$ \\
\hline Missouri & $1,270,000$ & $27.1(25.8-28.4)$ & $24.1(22.9-25.2)$ & 194,000 & $15.3(13.6-17.3)$ & $14.2(11.6-17.3)$ & 833,000 & $66.5(63.8-69.0)$ & $63.7(58.9-68.3)$ \\
\hline Montana & 241,000 & $28.9(27.7-30.2)$ & $25.4(24.3-26.6)$ & 37,000 & 15.7 (13.9-17.6) & $16.2(13.3-19.5)$ & 152,000 & $64.6(62.1-67.0)$ & $64.2(60.0-68.2)$ \\
\hline Nebraska & 335,000 & $23.1(22.3-24.0)$ & $21.0(20.2-21.7)$ & 51,000 & $15.4(14.0-16.9)$ & $14.6(12.2-17.4)$ & 223,000 & $67.2(65.3-69.1)$ & $64.7(60.9-68.4)$ \\
\hline Nevada & 531,000 & $22.7(20.6-25.0)$ & $20.7(18.7-22.8)$ & 96,000 & $18.2(14.3-22.9)$ & $15.4(11.7-20.2)$ & 366,000 & $69.0(63.7-73.8)$ & $70.2(61.9-77.4)$ \\
\hline New Har & 287,000 & $26.4(25.0-27.9)$ & $22.9(21.5-24.2)$ & 47,000 & $16.4(14.5-18.6)$ & $16.2(12.5-20.6)$ & 197,000 & $69.8(67.0-72.6)$ & $64.9(58.7-70.7)$ \\
\hline New Mexico & 413,000 & $25.8(24.4-27.3)$ & $23.2(21.9-24.5)$ & 75,000 & $18.1(15.8-20.6)$ & $18.8(15.1-23.2)$ & 295,000 & 71.7 (68.9-74.3) & $68.6(63.7-73.1)$ \\
\hline
\end{tabular}

See table footnotes on the next page.

attainment, urbanicity, federal poverty level, and joint pain severity. Groups with prevalences of self-management class attendance of $<15.0 \%$ included persons with a high school education or less $(12.8 \%)$; those employed (14.8\%), unemployed $(13.4 \%)$, or a student or homemaker $(12.8 \%)$; those residing in micropolitan (14.5\%) or rural areas (14.7\%); those who were inactive in the last 30 days (12.9\%); and those with no to mild joint pain (13.6\%). No differences in prevalence by sexual orientation or body mass index were observed.

Among adults with arthritis who reported having received counseling to be physically active, the median age-standardized prevalence was $69.3 \%$ (range $=59.9 \%$ [North Dakota] to $75.8 \%$ [Alaska]) (Table 1). The age-specific percentage of adults with arthritis who reported receipt of counseling was lowest among those aged 18-44 years (Table 2). Age-adjusted reporting of receipt of counseling was less prevalent among those physically inactive $(66.5 \%)$ in the last 30 days than among those active (73.1\%), among non-Hispanic American Indian or Alaska Native (67.8\%) or non-Hispanic White $(69.2 \%)$ persons than among Hispanic (75.3\%), or non-Hispanic Asian or Black persons $(75.1 \%$ and $76.0 \%$, respectively), and among those employed $(67.7 \%)$ or unemployed $(69.6 \%)$ than among those who were retired $(72.6 \%)$ or unable to work or disabled $(73.6 \%)$. Prevalence of receiving counseling increased with increasing education, urbanicity, body mass index, and joint pain severity. Groups among which $<67.0 \%$ had received counseling were men $(65.3 \%)$, those residing in rural areas $(66.0 \%)$, those who were inactive in the last 30 days $(66.5 \%)$, those who were underweight or healthy weight $(66.9 \%)$, and those who had no to mild joint pain $(66.3 \%)$. Prevalence of receiving physical activity counseling was similar across federal poverty level, marital status, and sexual orientation categories. No clear regional 
TABLE 1. (Continued) Unadjusted and age-standardized* prevalence of self-management education class attendance ${ }^{\dagger}$ and receipt of health care provider counseling about physical activity ${ }^{\S}$ among adults with arthritis ${ }^{\Uparrow}$ aged $\geq 18$ years — Behavioral Risk Factor Surveillance System, United States, ${ }^{* *} 2019$

\begin{tabular}{|c|c|c|c|c|c|c|c|c|c|}
\hline \multirow[b]{3}{*}{ Jurisdiction } & \multicolumn{3}{|c|}{ Persons with arthritis } & \multicolumn{3}{|c|}{$\begin{array}{l}\text { Self-management education } \\
\text { class attendance }\end{array}$} & \multicolumn{3}{|c|}{$\begin{array}{l}\text { Health care provider } \\
\text { physical activity counseling }\end{array}$} \\
\hline & \multirow[b]{2}{*}{ Est. no. ${ }^{\dagger \dagger}$} & \multicolumn{2}{|c|}{$\%(95 \% \mathrm{Cl})$} & \multirow[b]{2}{*}{ Est. no. ${ }^{\dagger \dagger}$} & \multicolumn{2}{|c|}{$\%(95 \% \mathrm{Cl})$} & \multirow[b]{2}{*}{ Est. no. ${ }^{\dagger \dagger}$} & \multicolumn{2}{|c|}{$\%(95 \% \mathrm{Cl})$} \\
\hline & & Unadjusted & $\begin{array}{c}\text { Age- } \\
\text { standardized }\end{array}$ & & Unadjusted & $\begin{array}{c}\text { Age- } \\
\text { standardized }\end{array}$ & & Unadjusted & $\begin{array}{c}\text { Age- } \\
\text { standardized }\end{array}$ \\
\hline $\mathrm{N}$ & & $22.1(21.2-23.0)$ & 1 & ) & ) & ) & 0 & 1) & 6 \\
\hline North Carolina & & $27.0(25.5-28.5)$ & $24.4(23.0-$ & 412,000 & $19.0(16.6-21.7)$ & $21.5(17.5-26.2)$ & 607,000 & $74.5(71.5-77.3)$ & $75.0(70.4$ \\
\hline North Dakota & 147,000 & $25.4(23.9-26.9)$ & $24.2(22.8-25.6)$ & 18,000 & $12.6(10.6-14.8)$ & $12.6(9.4-16.7)$ & 93,000 & $64.6(61.4-67.7)$ & $59.9(54.3-65.3)$ \\
\hline Ohio & $2,751,000$ & $30.6(29.5-31.8)$ & $27.5(26.4-28.6)$ & 422,000 & $15.4(13.9-17.1)$ & $15.5(13.2-18.2)$ & $1,926,000$ & $70.9(68.8-72.8)$ & $70.6(67.0$ \\
\hline Oklahoma & 000 & $27.0(25.7-28.3)$ & $25.0(23.9-26.2)$ &, 000 & $16.3(14.5-18.2)$ & $16.7(13.7$ & 2,000 & $67.1(64.5-69.6)$ & $65.0(60.4$ \\
\hline Oregon & 3,000 & $26.3(25.0-27.6)$ & $23.6(22.5-24.8)$ & 175,000 & $20.5(18.3-22.8)$ & $21.7(18.5$ & 605,000 & $71.4(68.7-74.0)$ & $69.2(65.1-$ \\
\hline Pennsylvania & $2,910,000$ & $29.1(27.7-30.5)$ & $25.1(24.0-26.3)$ & 372,000 & $12.8(11.2-14.7)$ & $12.7(10.0-$ & $2,031,000$ & $70.7(68.2-73.1)$ & $72.9(68.8$ \\
\hline Rhode Island & 224,000 & $26.8(25.3-28.3)$ & $23.8(22.5-25.2)$ & 33,000 & $14.9(12.9-17.0)$ & $15.3(11.6-20.0)$ & 168,000 & $75.7(73.0-78.2)$ & $75.5(69.4-80.6)$ \\
\hline South Carolina & $1,114,000$ & $28.2(26.9-29.5)$ & $25.0(23.8-26.3)$ & 172,000 & $15.5(13.7-17.4)$ & $13.6(11.2-16.5)$ & 760,000 & $68.8(66.2-71.2)$ & $64.7(60.0-69.1)$ \\
\hline South Dakota & 176,000 & $26.7(24.6-28.9)$ & $24.1(22.1-26.1)$ & 32,000 & $18.0(15.0-21.5)$ & $18.1(13.5-23.7)$ & 120,000 & $69.2(65.0-73.0)$ & $70.2(63.6-76.1)$ \\
\hline Tennessee & $1,598,000$ & $30.6(29.1-32.2)$ & $28.0(26.6-29.4)$ & 241,000 & $15.2(13.3-17.4)$ & $16.2(13.1-19.9)$ & $1,071,000$ & $67.9(65.2-70.6)$ & 66.5 (61.9-70.7) \\
\hline Texas & $4,398,000$ & $20.7(19.5-22.0)$ & $20.1(19.0-21.2)$ & 602,000 & $13.9(11.9-16.1)$ & $13.9(11.0-17.3)$ & $3,125,000$ & $72.0(68.9-74.9)$ & $69.4(64.0-74.2)$ \\
\hline Utah & 519,000 & $23.1(22.2-24.0)$ & $24.0(23.2-24.8)$ & 85,000 & $16.5(14.9-18.2)$ & $17.6(15.3-20.3)$ & 366,000 & $71.7(69.8-73.6)$ & $71.2(68.4-73.9)$ \\
\hline Vermont & 135,000 & $27.0(25.6-28.6)$ & $23.0(21.7-24.4)$ & 21,000 & $15.4(13.4-17.5)$ & $17.4(13.3-22.6)$ & 95,000 & $70.8(67.9-73.6)$ & $69.4(63.2-75.0)$ \\
\hline Virginia & $1,730,000$ & $26.3(25.2-27.4)$ & $24.0(23.0-25.1)$ & 286,000 & $16.6(14.9-18.5)$ & $17.7(14.6-21.1)$ & $1,206,000$ & $70.7(68.5-72.9)$ & $71.6(67.6-75.2)$ \\
\hline Washington & $1,439,000$ & $24.6(23.7-25.5)$ & $22.5(21.7-23.3)$ & 248,000 & $17.3(15.8-18.8)$ & $17.0(14.6-19.7)$ & $1,007,000$ & $70.8(69.0-72.6)$ & $71.5(68.3-74.4)$ \\
\hline West Virginia & 585,000 & $41.4(39.7-43.1)$ & $36.4(34.9-38.0)$ & 73,000 & $12.4(11.0-14.0)$ & $12.1(10.0-14.5)$ & 383,000 & $66.1(63.7-68.3)$ & $65.4(61.4-69.1)$ \\
\hline Wisconsin & $1,244,000$ & $27.8(26.3-29.3)$ & $24.6(23.3-26.0)$ & 196,000 & $15.8(13.7-18.1)$ & $19.7(15.3-25.0)$ & 880,000 & $71.6(68.8-74.2)$ & $74.3(69.6-78.5)$ \\
\hline Wyoming & 109,000 & $25.1(23.5-26.8)$ & $22.8(21.3-24.3)$ & 14,000 & $12.9(10.8-15.3)$ & $11.1(8.3-14.7)$ & 69,000 & $64.3(60.8-67.7)$ & $64.5(58.0-70.5)$ \\
\hline Guam & 17,000 & $16.1(14.0-18.5)$ & $17.7(15.6-20.0)$ & 3,000 & $16.3(12.5-21.0)$ & $17.2(12.2-23.6)$ & 12,000 & $72.7(64.3-79.8)$ & $66.8(57.0-75.3)$ \\
\hline Puerto Rico & 574,000 & $21.2(20.0-22.4)$ & $18.4(17.4-19.4)$ & 48,000 & $8.3(6.8-10.2)$ & $11.4(7.8-16.4)$ & 412,000 & $72.5(69.5-75.3)$ & $73.2(67.5-78.2)$ \\
\hline
\end{tabular}

Abbreviations: $\mathrm{Cl}=$ confidence interval; Est. = estimated; $\mathrm{NA}=$ not applicable.

* Estimates were age-standardized to the 2000 Projected U.S. Population aged $\geq 18$ years using three age groups: $18-44,45-64$, and $\geq 65$ years. https://www.cdc. gov/nchs/data/statnt/statnt20.pdf

† Respondents were classified as attending a self-management education course if they answered "yes" to the question, "Have you ever taken an education course or class to teach you how to manage problems related to your arthritis or joint symptoms?"

$\S$ Respondents were classified as receiving health care provider counseling to be physically active if they answered "yes" to the question, "Has a doctor or other health professional ever suggested physical activity or exercise to help your arthritis or joint symptoms?"

" Respondents were classified as having arthritis if they responded "yes" to the question, "Have you ever been told by a doctor or other health care professional that you have arthritis, rheumatoid arthritis, gout, lupus, or fibromyalgia?"

** In 2019, New Jersey did not collect enough data to meet the minimum requirement for inclusion in the Behavioral Risk Factor Surveillance System public-use data set.

${ }^{+t}$ Estimated number represents the weighted estimated number of adults with arthritis who reported the outcome of interest (e.g., health care provider counseling to be physically active and self-management education class attendance) rounded to the nearest thousand.

$\S \S$ Median calculated for 49 states and the District of Columbia.

patterns in the unadjusted and age-standardized prevalence of either self-management class attendance or counseling to be physically active were noted.

\section{Discussion}

The prevalence of self-management class attendance and receipt of health care provider counseling to be physically active among adults with arthritis varied considerably across states and by participant characteristics, with no clear regional patterns. Among adults with arthritis, self-management class attendance was low among all persons. The specific groups identified with low self-management class attendance and receipt of physical activity counseling were men, persons with a high school education or less, and those residing in small cities or rural areas. Opportunities for increasing health care provider counseling and interventions focused on improving self-management class attendance and physical activity among persons living with arthritis should continue for all, but especially for those groups with lower engagement in these activities.

The benefits of self-management courses and counseling to engage in physical activity are well established health goals for the nation, each of which was codified and evaluated in Healthy People 2020. The relevant Healthy People 2020 arthritis objective target*** of $11.7 \%$ of persons with arthritis attending self-management classes indicated slow progress and was almost attained in 2014 (11.4\%) as reported in the National Health Interview Survey (NHIS) (4). Similarly, advancement

\footnotetext{
*** Healthy People 2020 self-management education objective AOCBC-8, "Increase the proportion of adults with doctor-diagnosed arthritis who have had effective, evidence-based arthritis education as an integral part of the management of their condition." https://www.healthypeople.gov/2020/ topics-objectives/topic/Arthritis-Osteoporosis-and-Chronic-BackConditions/objectives
} 
TABLE 2. Overall, age-adjusted, and age-specific* prevalence of self-management education class attendance ${ }^{\dagger}$ and receipt of health care provider counseling for physical activity ${ }^{\S}$ among adults with arthritis aged $\geq 18$ years, ${ }^{\natural}$ by selected characteristics - Behavioral Risk Factor Surveillance System, United States, ${ }^{* *} 2019$

\begin{tabular}{|c|c|c|c|}
\hline \multirow[b]{2}{*}{ Characteristic } & \multirow[b]{2}{*}{$\begin{array}{l}\text { Unweighted } \\
\text { sample size }\end{array}$} & \multicolumn{2}{|c|}{$\%(95 \% \mathrm{Cl})$} \\
\hline & & $\begin{array}{l}\text { Self-management education } \\
\text { class attendance }\end{array}$ & $\begin{array}{l}\text { Health care provider counseling } \\
\text { for physical activity }\end{array}$ \\
\hline Overall (unadjusted) & 135,862 & $16.4(15.9-16.8)$ & $70.8(70.3-71.2)$ \\
\hline Overall (age-adjusted) & 135,862 & $16.3(15.9-16.7)$ & $70.8(70.3-71.3)$ \\
\hline \multicolumn{4}{|l|}{ Age-specific estimates } \\
\hline \multicolumn{4}{|l|}{ Age group, yrs ${ }^{t \dagger}$} \\
\hline $18-44$ & 11,665 & $16.9(15.7-18.1)$ & $67.9(66.4-69.4)$ \\
\hline $45-64$ & 47,991 & $16.4(15.8-17.1)$ & $71.2(70.4-71.9)$ \\
\hline$\geq 65$ & 76,206 & $16.1(15.5-16.7)$ & $71.4(70.8-72.1)$ \\
\hline \multicolumn{4}{|l|}{ Age-adjusted estimates } \\
\hline \multicolumn{4}{|l|}{ Sex } \\
\hline Female & 83,885 & $17.0(16.5-17.6)$ & $74.5(73.9-75.1)$ \\
\hline Male & 51,977 & $15.4(14.7-16.0)$ & $65.3(64.4-66.1)$ \\
\hline \multicolumn{4}{|l|}{ Race/Ethnicity } \\
\hline White, NH & 112,595 & $15.6(15.2-16.0)$ & $69.2(68.7-69.7)$ \\
\hline Black, NH & 10,407 & $18.1(16.8-19.5)$ & $76.0(74.5-77.5)$ \\
\hline Hispanic & 5,317 & $17.0(15.0-19.2)$ & $75.3(72.9-77.5)$ \\
\hline Asian, NH & 1,174 & $20.9(15.7-27.2)$ & $75.1(69.5-80.0)$ \\
\hline American Indian or Alaska Native, $\mathrm{NH}$ & 2,323 & $21.9(17.7-26.8)$ & $67.8(63.2-72.0)$ \\
\hline Other or multiple race, $\mathrm{NH}$ & 4,046 & $21.1(18.7-23.7)$ & $72.6(69.9-75.1)$ \\
\hline \multicolumn{4}{|l|}{ Marital status } \\
\hline Married & 67,122 & $16.0(15.5-16.6)$ & $70.7(70.0-71.4)$ \\
\hline Divorced, separated, or widowed & 52,525 & $17.3(16.6-18.1)$ & $70.4(69.6-71.2)$ \\
\hline Never married & 12,615 & $15.0(13.7-16.5)$ & $71.7(70.1-73.3)$ \\
\hline Member of an unmarried couple & 2,906 & $15.8(13.4-18.6)$ & $71.0(67.9-73.8)$ \\
\hline \multicolumn{4}{|l|}{ Highest level of education } \\
\hline Less than high school graduate & 10,894 & $12.8(11.5-14.1)$ & $67.2(65.6-68.8)$ \\
\hline High school graduate or equivalent & 39,281 & $12.8(12.1-13.5)$ & $69.2(68.3-70.1)$ \\
\hline Technical school or some college & 40,588 & $19.2(18.4-20.0)$ & $72.4(71.5-73.2)$ \\
\hline College degree or higher & 44,763 & $18.9(18.2-19.7)$ & $72.6(71.7-73.4)$ \\
\hline \multicolumn{4}{|l|}{ Employment status } \\
\hline Employed or self-employed & 42,601 & $14.8(14.1-15.5)$ & $67.7(66.8-68.6)$ \\
\hline Unemployed & 4,487 & $13.4(11.5-15.4)$ & $69.6(67.0-72.1)$ \\
\hline Retired & 62,828 & $17.6(16.7-18.5)$ & $72.6(71.6-73.5)$ \\
\hline Unable to work or disabled & 18,080 & $19.3(18.2-20.5)$ & $73.6(72.4-74.8)$ \\
\hline Other (student or homemaker) & 6,533 & $12.8(11.4-14.3)$ & $72.6(70.4-74.7)$ \\
\hline \multicolumn{4}{|l|}{ Federal poverty level $\$ \S$} \\
\hline$\leq 125 \% \mathrm{FPL}$ & 21,802 & $16.1(15.1-17.2)$ & $71.8(70.6-73.0)$ \\
\hline$>125 \%$ to $\leq 200 \% \mathrm{FPL}$ & 21,593 & $15.9(14.9-17.0)$ & $70.7(69.5-71.9)$ \\
\hline$>200 \%$ to $\leq 400 \% \mathrm{FPL}$ & 32,007 & $16.4(15.6-17.2)$ & $70.9(69.9-71.9)$ \\
\hline$>400 \% \mathrm{FPL}$ & 34,014 & $17.1(16.2-17.9)$ & $70.6(69.6-71.6)$ \\
\hline
\end{tabular}

See table footnotes on the next page.

toward the Healthy People 2020 arthritis objective target ${ }^{\dagger \dagger \dagger}$ of $57.4 \%$ of adults with arthritis receiving physical activity counseling indicated good progress and was surpassed in the 2014 NHIS, when $61.0 \%$ of adults with arthritis reported receiving such counseling (5).

Among the known benefits of physical activity for adults with arthritis are improved mood, strength, and endurance

\footnotetext{
t†† Healthy People 2020 health care provider counseling for physical activity objective AOCBC-7.2, "Increase the proportion of adults with doctordiagnosed arthritis who receive health care provider counseling for physical activity or exercise." https://www.healthypeople.gov/2020/topics-objectives/ topic/Arthritis-Osteoporosis-and-Chronic-Back-Conditions/objectives
}

and reduced arthritis-related joint pain, stiffness, and fatigue (6). Multiple professional organizations recommend that health care providers counsel adults with arthritis to engage in physical activity (7); however, a barrier commonly reported by providers is having insufficient training to counsel patients with arthritis (8). Health care providers can counsel patients about safely increasing physical activity using evidence-based, arthritis-appropriate, physical activity programs ${ }^{\$ \$ \$}$ available in communities across the country. These include low-impact group aquatic exercise (e.g., Arthritis Foundation Aquatic

\footnotetext{
\$S\$ https://www.cdc.gov/arthritis/interventions/physical-activity.html
} 
TABLE 2. (Continued) Overall, age-adjusted, and age-specific* prevalence of self-management education class attendance ${ }^{\dagger}$ and receipt of health care provider counseling for physical activity ${ }^{\S}$ among adults with arthritis aged $\geq 18$ years, " by selected characteristics — Behavioral Risk Factor Surveillance System, United States, ${ }^{* *} 2019$

\begin{tabular}{|c|c|c|c|}
\hline \multirow[b]{2}{*}{ Characteristic } & \multirow[b]{2}{*}{$\begin{array}{l}\text { Unweighted } \\
\text { sample size }\end{array}$} & \multicolumn{2}{|c|}{$\%(95 \% \mathrm{Cl})$} \\
\hline & & $\begin{array}{l}\text { Self-management education } \\
\text { class attendance }\end{array}$ & $\begin{array}{l}\text { Health care provider counseling } \\
\text { for physical activity }\end{array}$ \\
\hline \multicolumn{4}{|l|}{ Urban-rural status } \\
\hline Large central metro & 16,929 & $17.8(16.7-18.9)$ & $73.6(72.3-74.9)$ \\
\hline Large fringe metro & 23,940 & $16.1(15.2-16.9)$ & $71.4(70.3-72.4)$ \\
\hline Medium metro & 28,118 & $16.6(15.9-17.4)$ & $71.0(70.1-71.9)$ \\
\hline Small metro & 19,627 & $16.2(15.2-17.1)$ & $68.4(67.2-69.6)$ \\
\hline Micropolitan & 23,087 & $14.5(13.7-15.4)$ & $68.1(66.9-69.2)$ \\
\hline Rural (non-core) & 24,161 & $14.7(13.8-15.6)$ & $66.0(64.7-67.3)$ \\
\hline \multicolumn{4}{|l|}{ Sexual orientation ${ }^{* * *}$} \\
\hline Straight & 73,022 & $15.9(15.3-16.4)$ & $71.1(70.4-71.8)$ \\
\hline Lesbian, gay, bisexual, queer, or questioning & 4,264 & $15.5(13.0-18.5)$ & $72.3(69.5-75.0)$ \\
\hline \multicolumn{4}{|l|}{ Engaged in physical activity in past month ${ }^{++\dagger}$} \\
\hline Yes & 87,299 & $18.0(17.5-18.6)$ & $73.1(72.5-73.7)$ \\
\hline No & 42,960 & $12.9(12.3-13.6)$ & $66.5(65.6-67.4)$ \\
\hline \multicolumn{4}{|l|}{ Body mass index $\left(\mathrm{kg} / \mathrm{m}^{2}\right)$} \\
\hline Underweight or healthy weight $(<25)$ & 32,173 & $16.4(15.5-17.3)$ & $66.9(65.8-67.9)$ \\
\hline Overweight ( 25 to $<30)$ & 43,153 & $16.2(15.5-17.0)$ & $69.4(68.5-70.3)$ \\
\hline Obesity $(\geq 30)$ & 50,837 & $16.5(15.8-17.2)$ & $74.5(73.7-75.2)$ \\
\hline \multicolumn{4}{|l|}{ Joint pain severity $§ \S$} \\
\hline None/Mild & 62,913 & $13.6(13.0-14.2)$ & $66.3(65.5-67.0)$ \\
\hline Moderate & 32,184 & $17.8(16.9-18.7)$ & $74.7(73.7-75.7)$ \\
\hline Severe & 38,465 & $19.1(18.3-19.9)$ & $74.5(73.6-75.3)$ \\
\hline
\end{tabular}

Abbreviations: $\mathrm{Cl}=$ confidence interval; $\mathrm{FPL}=$ federal poverty level; $\mathrm{NH}=$ non-Hispanic.

* Except for the age groups category and the unadjusted overall variables, age-adjusted estimates were generated in weighted logistic regression models that included age as a categorical covariate using the following cut points: $18-44,45-64$, and $\geq 65$ years.

${ }^{\dagger}$ Respondents were classified as attending a self-management education course if they responded "yes" to the question, "Have you ever taken an education course or class to teach you how to manage problems related to your arthritis or joint symptoms?"

$\S$ Respondents were classified as receiving health care provider counseling to be physically active if they responded "yes" to the question, "Has a doctor or other health professional ever suggested physical activity or exercise to help your arthritis or joint symptoms?"

" Respondents were classified as having arthritis if they responded "yes" to the question, "Have you ever been told by a doctor or other health care professional that you have arthritis, rheumatoid arthritis, gout, lupus, or fibromyalgia?"

** In 2019, New Jersey did not collect sufficient data to meet the minimum requirement for inclusion in the Behavioral Risk Factor Surveillance System public-use data set.

t+ Age-specific estimates.

$\S \S$ Federal poverty level is the ratio of total family income to federal poverty guideline per family size.

१ๆ Urban-rural status was categorized using the National Center for Health Statistics 2013 Urban-Rural Classification Scheme for Counties. https://www.cdc.gov/ nchs/data/series/sr_02/sr02 166.pdf

*** Sexual orientation was asked in 30 states (Alaska, Arizona, Colorado, Connecticut, Delaware, Florida, Georgia, Hawaii, Idaho, lowa, Kansas, Louisiana, Maryland, Minnesota, Mississippi, Montana, New York, North Carolina, Ohio, Oklahoma, Rhode Island, South Carolina, Tennessee, Texas, Utah, Vermont, Virginia, Washington, West Virginia, and Wisconsin).

tt+ Physical activity was defined using the question, "During the past month, other than your regular job, did you participate in any physical activities or exercises such as running, calisthenics, golf, gardening, or walking for exercise?"

$\S \S \S$ For the question, "On a scale of 0 to 10 where 0 is no pain or aching and 10 is pain or aching as bad as it can be, during the past 30 days, how bad was your joint pain on average," an answer of 0-4 was defined as none or mild, an answer of 5-6 was defined as moderate, and an answer of 7-10 was defined as severe.

Program); EnhanceFitness, which incorporates balance activities; Fit and Strong!, which emphasizes flexibility, strength training, aerobic walking and health education to promote behavior change; and Walk with Ease, which combines selfpaced walks with instruction on health-related topics and can be delivered as a group or self-directed activity, both of which accommodate physical distancing, as recommended during the COVID-19 pandemic.

Recommending self-management class attendance while counseling persons with arthritis to engage in physical activity might be the most effective strategy for increasing physical activity. A health care provider's recommendation to attend a self-management workshop is strongly associated with selfmanagement class attendance (9). A meta-analysis of health outcomes, health behaviors, and health care utilization related to self-management programs found that persons with arthritis who received a health care provider recommendation to attend a self-management class were nine times more likely to attend a class than were those who did not receive a recommendation (10). The analysis found that aerobic physical activity increased after attendance in the generic, evidence-based self-management 


\section{Summary}

What is already known about this topic?

Arthritis is a prevalent chronic condition. Self-management education and health care provider counseling encouraging engagement in physical activity can improve the health of adults with arthritis; however, in 2014 , only $11.4 \%$ and $61.0 \%$ of arthritis patients reported engaging in each, respectively.

What is added by this report?

In 2019 , a median of $16.2 \%$ adults with arthritis attended a self-management class, and $69.3 \%$ received provider counseling for physical activity. Prevalences differed by state and sociodemographic characteristics.

What are the implications for public health practice?

Equipping health care providers with the tools to counsel arthritis patients about the benefits of physical activity and self-management education and support referrals to evidence-based programs is needed to improve adoption of these behaviors.

course99 (Chronic Disease Self-Management Program [CDSMP]) and persisted for 1 year after attending the class (10). CDSMP is a workshop tailored to adults with chronic conditions (including arthritis) and other comorbidities which are also common among adults with arthritis (1); the workshop teaches improved self-efficacy and skills, resulting in better arthritis outcomes. Benefits of CDSMP include improved health status (e.g., reduced pain, and improved function and psychological health), improved health behaviors (e.g., increased physical activity, and improved healthful eating, pain-coping strategies, and medication adherence), and improved communication with health care providers. CDSMP is offered in a Spanish-language version (Tomando Control de su Salud) and virtually by the Better Choices, Better Health program.

The findings in this report are subject to at least three limitations. First, BRFSS data rely on self-report and might be subject to recall, social desirability, and other biases. Second, low response rates that differ by state might bias study findings; however, the weighting methodology accounts for nonresponse. Finally, the question to ascertain self-management class attendance did not establish whether respondents attended an evidence-based self-management course. A strength of this study is the use of recent data with a large sample size that allowed analyses of detailed characteristics in 49 states, DC, and two U.S. territories. In addition, the prevalence estimates generated are representative at the state level.

Self-management class attendance and health care provider counseling for physical activity varied by state and sociodemographic characteristics among adults with arthritis. Public health professionals and medical groups can help improve

\footnotetext{
999 https://www.cdc.gov/arthritis/interventions/self_manage.htm
}

patient self-management behaviors and outcomes among patients with arthritis by equipping health care providers**** with the tools and information they need to counsel adults with arthritis to be active and recommend evidence-based physical activity and self-management programs.

**** https://www.cdc.gov/arthritis/healthcare/index.html

Corresponding author: Lindsey M. Duca, 404-498-2798,pgz5@cdc.gov.

\footnotetext{
${ }^{1}$ Epidemic Intelligence Service, CDC; ${ }^{2}$ Division of Population Health, National Center for Chronic Disease Prevention and Health Promotion, CDC; ${ }^{3} \mathrm{Oak}$ Ridge Institute for Science and Education, Oak Ridge, Tennessee.
}

All authors have completed and submitted the International Committee of Medical Journal Editors form for disclosure of potential conflicts of interest. No potential conflicts of interest were disclosed.

\section{References}

1. Barbour KE, Helmick CG, Boring M, Brady TJ. Vital signs: prevalence of doctor-diagnosed arthritis and arthritis-attributable activity limitation-United States, 2013-2015. MMWR Morb Mortal Wkly Rep 2017;66:246-53. PMID:28278145 https://doi.org/10.15585/ mmwr.mm6609e1

2. Barbour KE, Boring M, Helmick CG, Murphy LB, Qin J. Prevalence of severe joint pain among adults with doctor-diagnosed arthritisUnited States, 2002-2014. MMWR Morb Mortal Wkly Rep 2016;65:1052-6. PMID:27711038 https://doi.org/10.15585/mmwr. mm6539a2

3. CDC. Arthritis: lifestyle management programs. Atlanta, GA: US Department of Health and Human Services, CDC; 2021. https://www. cdc.gov/arthritis/interventions/index.htm

4. Murphy LB, Brady TJ, Boring MA, et al. Self-management education participation among U.S. adults with arthritis: who's attending? Arthritis Care Res (Hoboken) 2017;69:1322-30. PMID:27748081 https://doi. org/10.1002/acr.23129

5. Hootman JM, Murphy LB, Omura JD, et al. Health care provider counseling for physical activity or exercise among adults with arthritisUnited States, 2002 and 2014. MMWR Morb Mortal Wkly Rep 2018;66:1398-401. PMID:29300722 https://doi.org/10.15585/mmwr. mm665152a2

6. 2018 Physical Activity Guidelines Advisory Committee. 2018 Physical Activity Guidelines Advisory Committee scientific report. Washington, DC: U.S. Department of Health and Human Services; 2018. https://health.gov/ sites/default/files/2019-09/PAG_Advisory_Committee_Report.pdf

7. Kolasinski SL, Neogi T, Hochberg MC, et al. 2019 American College of Rheumatology/Arthritis Foundation guideline for the management of osteoarthritis of the hand, hip, and knee. Arthritis Rheumatol 2020;72:220-33. PMID:31908163 https://doi.org/10.1002/art.41142

8. Lillie K, Ryan S, Adams J. The educational needs of nurses and allied healthcare professionals caring for people with arthritis: results from a cross-sectional survey. Musculoskelet Care 2013;11:93-8. PMID:23065861 https://doi.org/10.1002/msc.1035

9. Murphy LB, Theis KA, Brady TJ, Sacks JJ. Supporting self-management education for arthritis: Evidence from the Arthritis Conditions and Health Effects Survey on the influential role of health care providers. Chronic Illn 2021;17:217-31. PMID:31475576 https://doi. org/10.1177/1742395319869431

10. Brady TJ, Murphy L, O'Colmain BJ, et al. A meta-analysis of health status, health behaviors, and health care utilization outcomes of the Chronic Disease Self-Management Program. Prev Chronic Dis 2013;10:120112. PMID:23327828 https://doi.org/10.5888/ $\operatorname{pcd} 10.120112$ 